\title{
GADISI - Genetic Algorithms Applied to the Automatic Design of Integrated Spiral Inductors
}

\author{
Pedro Pereira, M. Helena Fino, Fernando Coito, and Mário Ventim-Neves \\ Faculdade de Ciências e Tecnologia - Universidade Nova de Lisboa, \\ 2829-516 Caparica, Portugal \\ pmrp@fct.unl.pt, hfino@ieee.org, fjvc@fct.unl.pt, \\ ventim@uninova.pt
}

\begin{abstract}
This work introduces a tool for the optimization of CMOS integrated spiral inductors. The main objective of this tool is to offer designers a first approach for the determination of the inductor layout parameters. The core of the tool is a Genetic Algorithm (GA) optimization procedure where technology constraints on the inductor layout parameters are considered. Further constraints regarding inductor design heuristics are also accounted for. Since the layout parameters are inherently discrete due to technology and topology constraints, discrete variable optimization techniques are used. The Matlab GA toolbox is used and the modifications on the GA functions, yielding technology feasible solutions is presented. For the sake of efficiency and simplicity the pi-model is used for characterizing the inductor. The validity of the design results obtained with the tool, is checked against circuit simulation with ASITIC.
\end{abstract}

Keywords: Discrete-Variable Optimization, Genetic Algorithm, Spiral Inductor Design.

\section{Introduction}

The rapid growth and reduced cost of communication systems operating at high frequencies, has motivated the development of devices with a large number of elements implemented in integrated circuit. In the particular case of RF transceivers, voltage controlled oscillators (VCOs) are responsible for the purity of the signal generated. For these applications LC-VCOs are often chosen due to their phase-noise behaviour. The performance of the LC-VCOs is well determined by the quality factor of the spiral inductor.

For the design of spiral inductors, designers usually adopt methodologies based on either pre-characterized inductor designs or ad hoc techniques [1]. In either case designing a spiral inductor involves the determination of multiple correlated variables. Due to the complexity of the design, optimization-based techniques have been proposed [2], [3] and [4]. In these approaches, however, continuous variable optimization techniques are employed and constraints are considered only on the bounding values for the inductor layout parameters. Technology-constrains regarding the discrete nature of the variables is dealt in a subsequent procedure where the layout 
parameters obtained from optimization are rounded to the nearest technology feasible value. This methodology usually yields sub-optimum solutions where the correlation of the parameters obtained is not granted.

During the last years GAs have been widely used in circuit design [5] and [6]. This paper introduces a GA tool for the optimization-based design of spiral inductors. In this tool the solution is obtained considering user-defined constraints in the design, reflecting a discretization of the variable values according to the technology used. Further constraints imposing bounds on the ratio between design variables are also accounted for as a way of supporting design heuristics. The tool offers the possibility to choose the performance parameter to be optimized, such as maximizing the quality factor, $Q$, at a given operation frequency, or the minimization of the area occupied by the device. The tool was developed in Matlab and the GA optimization toolbox was used. The validity of the solution obtained is checked against results from simulation with ASITIC simulator.

\section{Contribution to Technological Innovation}

The main objective of the optimization based tool presented is to generate the geometrical layout parameters of integrated spiral inductors. For this purpose two main concerns were considered. On one hand a technology-aware methodology is adopted where the discrete nature of the variables is accounted for as a way of restricting the search space to those points allowed by the technology. Further constraints arising from heuristic design rules are also considered. On the other hand the necessity for obtaining solutions in an efficient way led to the use of the inductor physical model, instead of using a simulation based approach.

\subsection{Planar Spiral Inductor Pi-Model}

The efficiency of the optimization tool is obtained through the use of an inductor model. For the sake of simplicity the pi-model, illustrated in Fig. 1.a., is adopted.

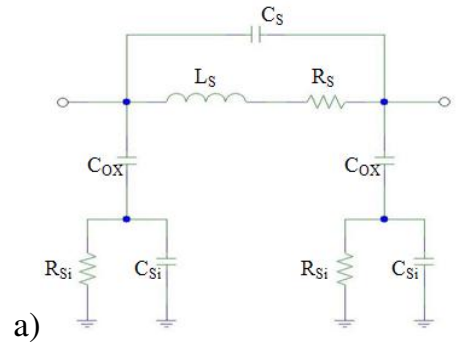

Fig. 1. a) Planar inductor pi-model; b) Layout of a square inductor

For the evaluation of the inductance, $L s$, several approaches have been proposed, based on a fitting process to values obtained experimentally [7], or through physicsbased equations [8]. In this tool the Modified Wheeler formula is used [7], where 


$$
\mathrm{L}_{\mathrm{s}}=\mathrm{k}_{1} \mu_{0} \mathrm{n}^{2} \mathrm{~d}_{\mathrm{avg}} /\left(1+\mathrm{k}_{2} \rho\right) \text {, }
$$

given that

$$
\begin{aligned}
& \rho=\left(d_{\text {out }}-d_{\text {in }}\right) /\left(d_{\text {out }}-d_{\text {in }}\right), \\
& d_{\text {avg }}=0.5\left(d_{\text {out }}+d_{\text {in }}\right), \\
& d_{\text {out }}=d_{\text {in }}+2 n w+2(n-1) \mathrm{s} .
\end{aligned}
$$

where, $n$ is the number of turns, $s$ is the track to track distance, and $w$ is the track width, as represented in Fig. 1.b. Finally, $k_{l}$ and $k_{2}$, are coefficients allowing the model to be adapted to several inductor shapes [7]. The evaluation of $\mathrm{R}_{\mathrm{s}}, \mathrm{R}_{\mathrm{si}} \mathrm{C}_{\mathrm{s}}, \mathrm{C}_{\mathrm{ox}}$, and $\mathrm{C}_{\mathrm{si}}$, is obtained with equations in [9] and [10].

\subsection{Genetic Algorithms Applied to Integrated Inductor Design}

Genetic Algorithms (GAs) are a stochastic search method that mimics the natural biological evolution, operating on a population of potential solutions, applying the principle of survival of the fittest to produce better and better approximations to a solution. GAs can be applied to any problem that can be formulated as function optimization problems. As a result, they are applied to non-linear problems, defined on discrete, continuous or mixed search spaces, constrained or unconstrained. Besides that, GAs are conceptually simple and have proved to be robust to dynamic changes.

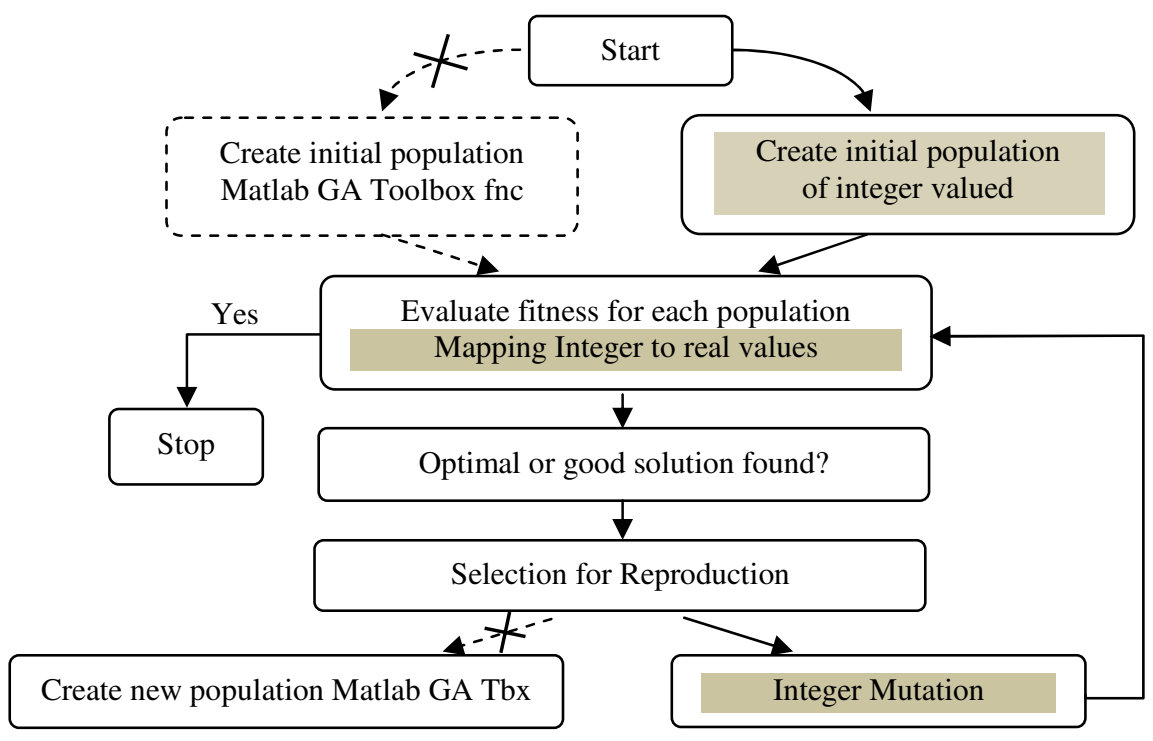

Fig. 2. Simple Genetic Algorithm flowchart 
The main advantages of using GAs in optimization problems instead of the more traditional search and optimization methods reside in the fact that they search a population of points in parallel instead of a single point, thus making results less sensitive to the point chosen. Furthermore, GAs do not require derivative information or previous knowledge, they handle noisy functions well, and are resistant to becoming trapped in local optima [11].

The optimization tool developed was implemented with the Matlab GA toolbox. Since this toolbox shows several limitations for discrete optimization problems, where each variable has an imposed step-size, three functions were developed. The flowchart showing the process of GA is represented in Fig. 2, where the additional functions, are shown with a dark background.

\section{Optimization Methodology for Inductor Design}

The methodology adopted aims to achieve a technology/topology-aware solution, where constraints are mandatory. Regarding the number of turns, $n$, it was considered to have a minimum value of 1.5 and subsequent values are obtained with an unitary increment, i.e.,

$$
\mathrm{n} \in[1.5,2.5,3.5, \ldots . .[
$$

Due to the inductor model considered the shape of the inductor must account for square, hexagonal and octagonal topologies.On what concerns the technological constraints, minimum values for the track width, $w$, for the track-to-track spacing, $s$, and for in input diameter, $d_{i n}$, must be defined. Technology-depend minimum increment values for these layout parameters must also be considered. Finally the correlation between the layout parameters defined by (4) is considered, as a way of including heuristic design rules for reducing the parasitic phenomena due to the proximity effect [12].

$$
0.2<\mathrm{d}_{\text {in }} / \mathrm{d}_{\text {out }}<0.8, \mathrm{~d}_{\text {in }}>5 \mathrm{w} .
$$

If we define $\operatorname{Cost}\left(\mathrm{n}, \mathrm{d}_{\mathrm{in}}, \mathrm{w}, \mathrm{N}\right)$ and $\mathrm{L}\left(\mathrm{n}, \mathrm{d}_{\mathrm{in}}, \mathrm{w}, \mathrm{N}\right)$ as the cost function and the inductance of the spiral, and $\mathrm{L}_{\text {exp }}$ and $\delta$ the targeting inductance value and the tolerance allowed for the inductance to deviate from the targeting value, the optimization problem is formulated as the minimization of $\operatorname{Cost}\left(\mathrm{n}, \mathrm{d}_{\mathrm{in}}, \mathrm{w}, \mathrm{N}\right)$ subject to

$$
\begin{gathered}
(1-\delta) \mathrm{L}_{\text {exp }} \leq \mathrm{L}\left(\mathrm{n}, \mathrm{d}_{\mathrm{in}}, \mathrm{w}, \mathrm{N}\right) \leq(1+\delta) \mathrm{L}_{\text {exp }} \\
\mathrm{w} \in\left[\mathrm{w}_{\text {min }}: \operatorname{step}_{\mathrm{w}}: \mathrm{w}_{\max }\right] \\
\mathrm{d}_{\mathrm{in}} \in\left[\mathrm{d}_{\text {min }}: \operatorname{step}_{\mathrm{d}}: \mathrm{d}_{\text {max }}\right] \\
\mathrm{n} \in\left[\mathrm{n}_{\text {min }}: \operatorname{step}_{\mathrm{n}}: \mathrm{n}_{\text {max }}\right] \\
\mathrm{N} \in[2,4,8]
\end{gathered}
$$

Concerning the cost function three different scenarios are available yielding either the minimization of the tolerance, $\delta$, the minimization of the device area, $\mathrm{d}_{\text {out }}$, or the maximization of the quality factor, for a predefined frequency of operation and a 
maximum output diameter, $\mathrm{d}_{\text {out }}$ [13]. For the pi-model, the expression of the quality factor becomes [14]

$$
\mathrm{Q}=\left(\omega \mathrm{L}_{\mathrm{s}} / \mathrm{R}_{\mathrm{s}}\right) \cdot\left(\mathrm{R}_{\mathrm{p}} /\left(\mathrm{R}_{\mathrm{p}}+\left[\left(\frac{\omega \mathrm{L}_{\mathrm{s}}}{\mathrm{R}_{\mathrm{s}}}\right)^{2}+1\right] \mathrm{R}_{\mathrm{s}}\right)\right) \cdot\left(1-\left(\mathrm{C}_{\mathrm{p}} \mathrm{R}_{\mathrm{s}}^{2} / \mathrm{L}_{\mathrm{s}}\right)-\omega^{2} \mathrm{~L}_{\mathrm{s}} \mathrm{C}_{\mathrm{p}}\right) .
$$

Where

$$
\begin{gathered}
\mathrm{R}_{\mathrm{p}}=\left(1 / \omega^{2} \mathrm{C}_{\mathrm{ox}}^{2} \mathrm{R}_{\mathrm{si}}\right)+\left(\mathrm{R}_{\mathrm{si}}\left(\mathrm{C}_{\mathrm{ox}}+\mathrm{C}_{\mathrm{si}}\right)^{2} / \mathrm{C}_{\mathrm{ox}}^{2}\right) \\
\mathrm{C}_{\mathrm{p}}=\mathrm{C}_{\mathrm{ox}}\left(1+\omega^{2}\left(\mathrm{C}_{\mathrm{ox}}+\mathrm{C}_{\mathrm{si}}\right) \mathrm{C}_{\mathrm{si}} \mathrm{R}_{\mathrm{si}}^{2}\right) / 1+\omega^{2}\left(\mathrm{C}_{\mathrm{ox}}+\mathrm{C}_{\mathrm{si}}\right)^{2} \mathrm{R}_{\mathrm{si}}^{2}
\end{gathered}
$$

For the inductor design, the GA procedure starts with the creation of the initial population, where each individual is composed by four variables $(\mathrm{w}, \mathrm{d}, \mathrm{n}, \mathrm{N})$, representing the layout geometry parameters. The initial population is randomly created, but the individual's genes must obey to variable boundaries. Represented in Fig. 2 an alternative functions for creating an integer-valued population is used.

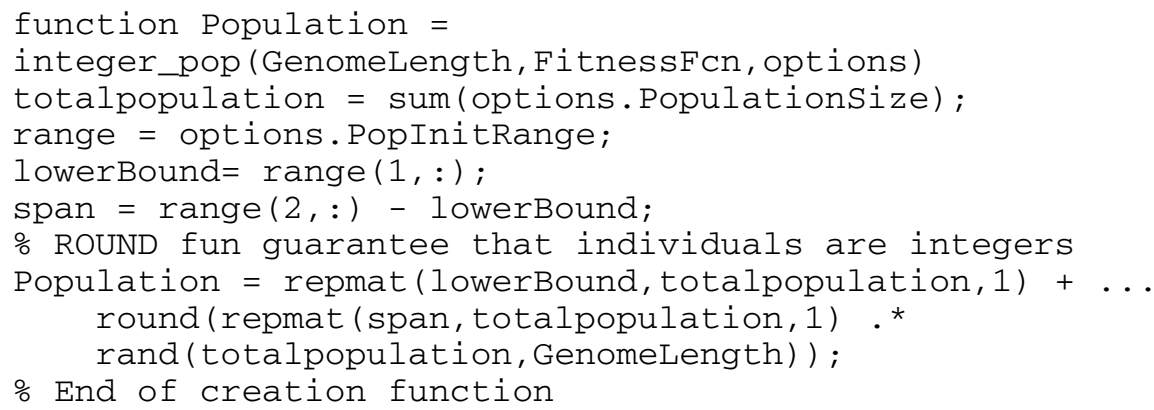

The population is converted to real values and then evaluated through the objective function. In this function each individual is evaluated according to the constraints imposed and if it is not compliant, a penalty is applied so that it shows a very low probability of being elected for the next population. The algorithm stops here, if termination conditions are verified.

The next step accounts for the generation of a new population. Here selection and reproduction functions are used. For the selection the roulette wheel method is chosen. Afterwards, reproduction (or mutation) is made. Due to the difficulty of Matlab in dealing with real-valued population with predefined step-size, a mutation function was defined, allowing the creation of a new population of integer-valued individuals.

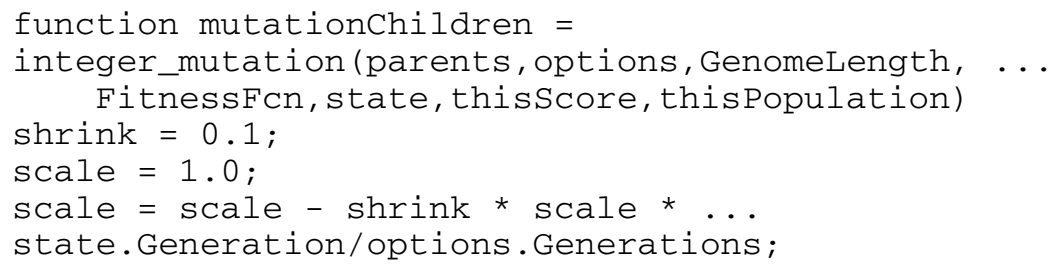




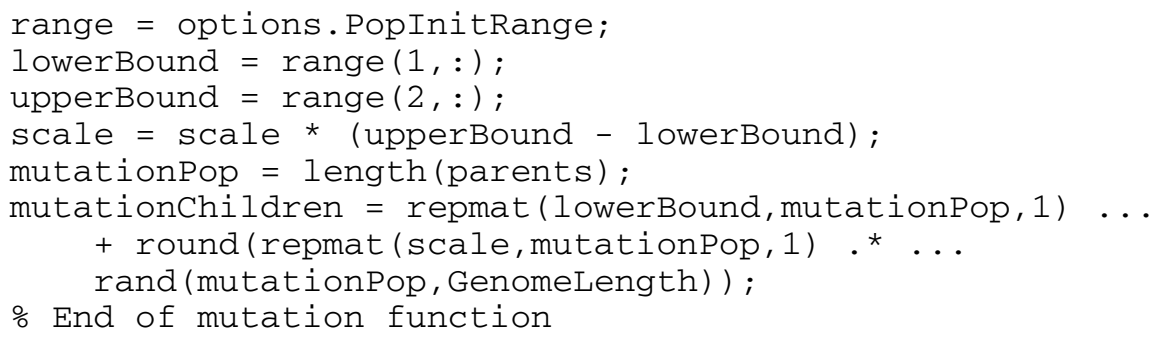

\section{Results}

The results presented in this section spotlight the ability of the developed GA-based tool to achieve optimal design of spiral inductors. The design of $7 \mathrm{nH}$ inductor for an operation frequency of $0.8 \mathrm{GHz}$ is addressed, where the technological parameters shown in Table 1 are used to evaluate $\mathrm{R}_{\mathrm{si}}, \mathrm{C}_{\mathrm{s}}, \mathrm{C}_{\mathrm{ox}}$ and $\mathrm{C}_{\mathrm{si}}$. The determination of the layout parameters is done according to the constraints represented in Table 2. Also a minimum space between tracks of $1.5 \mu \mathrm{m}$ and a maximum tolerance $\delta$ of $2.5 \%$, are assumed.

In this section, three examples are addressed. In example $A$, the inductor layout parameters minimizing the tolerance, $\delta$, are computed; example $B$ deals with designing an inductor with the smallest device area. Finally in example $C$ an inductor with maximum quality factor, given a maximum output diameter of $500 \mu \mathrm{m}$ is obtained. In examples $B$ and $C$ the tolerance, $\delta$, is an additional constraint, with maximum value of $2.5 \%$. The results obtained are presented in Table 3 .

Table 1. Technological parameters

\begin{tabular}{cccc}
\hline Parameter & Value & Parameter & Value \\
\hline$\varepsilon_{o}$ & $8.85 \mathrm{e}-12$ & $t_{o x}(\mu \mathrm{m})$ & 600 \\
$\varepsilon_{r}$ & 1.0 & $C_{\text {sub }}\left(\mathrm{F} / \mathrm{m}^{2}\right)$ & $4.0 \mathrm{e}-6$ \\
$\sigma(\Omega \mathrm{m})$ & $1 / 2.65 \mathrm{e}-8$ & $G_{s u b}\left(\mathrm{~S} / \mathrm{m}^{2}\right)$ & $2.43 \mathrm{e}+5$ \\
\hline
\end{tabular}

Table 2. Design Constraints

\begin{tabular}{cccc}
\hline Parameter & Min & Step & Max \\
\hline$w(\mu \mathrm{m})$ & 5.0 & 0.25 & 50.0 \\
$d_{\text {in }}(\mu \mathrm{m})$ & 20.0 & 0.25 & 250.0 \\
$n$ & 1.5 & 1.0 & 15.5 \\
\hline
\end{tabular}

Regarding the results in Table 3, for the working example $A$, we should mention that several different results can be obtained for the same goal. This means that there is a grid of solutions that match the objective. On the other hand for examples $B$ and $C$ the use of a maximum tolerance, $\delta$, as an additional restriction, leads to the 
Table 3. Optimization results

\begin{tabular}{ccccccccc}
\hline & $\mathrm{w}(\mu \mathrm{m})$ & $\mathrm{d}_{\text {in }}(\mu \mathrm{m})$ & $\mathrm{n}$ & $\mathrm{N}_{\text {side }}$ & $\mathrm{d}_{\text {out }}(\mu \mathrm{m})$ & $\mathrm{L}_{\text {tool }}(\mathrm{nH})$ & $\delta(\%)$ & $\mathrm{Q}_{\text {tool }}$ \\
\hline$A$ & 11.00 & 149.50 & 4.5 & 4 & 259.00 & 7.00 & 0.0 & 3.90 \\
$B$ & 5.50 & 48.50 & 7.5 & 4 & 150.50 & 6.83 & 2.42 & 2.35 \\
$C$ & 34.75 & 176.25 & 4.5 & 8 & 499.50 & 7.17 & 2.42 & 9.19 \\
\hline
\end{tabular}

Table 4. Comparison of results between evaluated and simulated

\begin{tabular}{ccccccc}
\hline & $\mathrm{L}_{\text {tool }}(\mathrm{nH})$ & $\mathrm{L}_{\text {Asitic }}(\mathrm{nH})$ & Error $(\%)$ & $\mathrm{Q}_{\text {tool }}(\mathrm{nH})$ & $\mathrm{Q}_{\text {Asitic }}(\mathrm{nH})$ & Error $(\%)$ \\
\hline$A$ & 7.00 & 6.97 & 0.43 & 3.90 & 3.83 & 1.82 \\
$B$ & 6.83 & 6.73 & 1.49 & 2.35 & 2.30 & 2.17 \\
$C$ & 7.17 & 7.01 & 2.23 & 9.19 & 8.65 & 6.24 \\
\hline
\end{tabular}

optimum result shown. It is also possible to conclude that there is a commitment between $\mathrm{Q}$ and $\mathrm{d}_{\text {out }}$,i.e., when maximizing $\mathrm{Q}$, the largest area is chosen. The validity of these results was checked against simulation with ASITIC yielding results shown in Table 4.

\section{Conclusions}

This paper introduces a tool for the determination of the layout parameters of CMOS spiral inductors. In this tool a GA optimization methodology is adopted, where constraints on the values of the variables representing the layout parameters are used. These constraints regard not only a limitation on the maximum/minimum values of the variables but also a discretization on their values. This enables the generation of the best solution for the technology used. Further constraints imposing predefined correlation between layout parameters are considered as a way of implementing heuristic design rules. Three working examples showing the design of an inductor for different optimization goals were shown. The solutions obtained are in good accuracy when compared to simulation results, with errors below $7 \%$.

The evolution of the tool to a multi-objective optimization will be addressed, allowing designers to have a better understanding of the solution space, and to choose the best solution. The main limitation of the work proposed resides in the applicability of the tool to higher frequencies. This limitation is inherent to the pi-model used for the integrated inductor. The inclusion of more appropriate high frequency model will be considered as a continuation of the work proposed.

\section{References}

1. Hamm, D.: Design of Integrated LC VVCOs. In: Toumazou, C., Moschytz, G., Gilbert, B. (eds.) Trade-offs in Analog Circuit Design, ch. 18, pp. 517-589. Kluwer Academic Publishers, Dordrecht (2002)

2. Nieuwoudt, A., Massoud, Y.: Multi-level Approach for Integrated Spiral Inductor Optimization. In: Design Automation Conference, pp. 648-651 (2005) 
3. Allstot, D., Choi, K., Park, J.: Parasitic-Aware Optimization of CMOS RF Circuits. Kluwer Academic Publishers, Dordrecht (2003)

4. Chan, T., Lu, H., Zeng, J., Chen, C.: LTCC Spiral Inductor Modeling, Synthesis and Optimization. In: ASPDAC 2008, pp. 768-771 (2008)

5. Stelmack, M., Nakashima, N., Batill, S.: Genetic Algorithms for Mixed Discrete/ Continuous Optimization in Multidisciplinary Design. In: Symposium on Multidisciplinary Analysis and Optimization (1998)

6. Wankhede, M., Deshmukh, A.: Optimization of Cell-based VLSI Circuit Design using a Genetic Algorithm: Design Approach. In: Proceedings of the International MultiConference of Engineers and Computer Scientists (2009) ISBN 978-988-17012-7-5

7. Mohan, S., Hershenson, M., Boyd, S., Lee, T.: Simple Accurate Expressions for Planar Spiral Inductances. IEEE Journal of Solid-State Circuits 34 (1999)

8. Jenei, S., Nauwelaers, B., Decoutere, S.: Physics-Based Closed-Form Inductance Expression for Compact Modelling of Integrated Spiral Inductors. IEEE J. Solid-State Circuits 37, 77-80 (2002)

9. Choi, Y., Yoon, J.: Experimental Analysis of the Effect of Metal Thickness on the Quality Factor in Integrated Spiral Inductors for RF ICs. IEEE Electron Device Lett. 25, 76-79 (2004)

10. Murphy, O.: Advanced Physical Modelling of Multilayer Inductors for CMOS RF FrontEnd Applications. PhD Thesis, University College Cork - National University of Ireland (2005)

11. Sivanandam, S., Deepa, S.: Introduction to Genetic Algorithms. Springer, Heidelberg (2008)

12. Aguilera, J., Berenguer, R.: Design and Test of Integrated Inductors for RF Applications. Kluwer Academic Publishers, Dordrecht (2004)

13. Pereira, P., Fino, M.H., Coito, F., Ventim-Neves, M.: ADISI - An efficient tool for the automatic design of integrated spiral inductor. In: 16th IEEE International Conference on Electronics, Circuits and Systems - IEEE ICECS 2009, Hammamet - Tunisia (December 2009) (accepted for publication)

14. Yue, C., Wong, S.: Design Strategy of On-Chip Inductors for Highly Integrated RF Systems. In: Proceedings of the 1999 Design Automation Conference, pp. 982-987 (1999) 